ostensible indication and evidence of the changes that are taking place in the ovaries.

It is now universally admitted that the menstrual secretion takes place from the mucous membrane lining the uterine cavity. For one or two days before it commences, in the healthy uterus, a tide of blood sets in towards the uterine organs; and if the cervix uteri is then brought into view, its mucous surface is found greatly congested, and of a livid hue. When the secretion has commenced, the blood may be seen to ooze guttatim from the os uteri. After it has ceased, the tide of blood gradually recedes, and in the course of one, two, or three days, the uterus is restored to its normal condition, the cervix assuming its naturally pale, rosy hue. If the uterus is the seat of disease, the flux to it begins earlier - often a week before. After menstruation has ceased, there is also, in disease, a great tendency to the perpetuation of the menstrual congestion, the uterus frequently not appearing to have the power to expel the menstrual blood.

Menstruation in the human female oscillates physiologically between great extremes, or, in other words, it may vary to an extreme extent in its mode of manifestation, and yet these variations may be compatible with health, and with the perfect integrity of the uterine organs. Indeed, there is not a greater difference between the human female and the female of the lower mammiferæ, in which the menstrual function only shows its presence by a congested state of the genital organs and a slight mucous secretion, than there is bet ween different females. Thus, for instance, in some, the menstrual flux only shows itself for a day or two, or even for a few hours, throughout life, and is very seanty ; whereas, in others, it lasts seven or eight days, and is always so profuse as to be all but hæmorrhagic.

The physiological variations of menstruation may be referred to its epoch of first manifestation, to its duration, to the quantity of blood lost, to the amount of pain experienced, and to the periodicity of its return.

The epoch at which menstruation first sets in, is very variable, but may be said to range between eleven and nineteen or twenty, the cases in which it occurs before or after these ages being rare. The medium age, in temperate climates, according to Raciborski, who deduced it from the analysis of a large number of cases, is about fourteen - a statement which my own experience comple ely corroborates. There are cases on record, in which menstruation has set in as early even as the third or fourth year, but they can merely be considered freaks of nature. Climate was formerly considered to exercise great influence over the epoch at which menstruation appears, but this influence appears to have been greatly exaggerated. So far from cold greatly retarding, and heat greatly accelerating, its appearance, it would appear, from the valuable researches of Dr. Roberton, ${ }^{*}$ of Manchester, that the medium age is pretty nearly the same all over the world. Raciborski finds a difference in the medium age of the cases he investigated for the north and south of France, but that difference only amounts to a few months, and would require to be deduced from a larger number of persons, to be definitively accepted. Menstruation generally ceases between forty-five and fifty, but the menopause may occur much earlier or much later.

The duration of the menstrual flax, and the quantity of blood lost, vary very considerably in different females. The average duration may be said to be about four or five days, but many are only unwell two or three, and with many again, it lasts six or seven. When menstruation is of short duration, the loss of blood is generally scanty, whereas it is greater when it lasts a long period; not only on account of its longer duration, but also because it generally flows more freely. The influence of climate in this respect also appears to have been much exaggerated. The fact of menstruation being constitutionally of long duration and profuse, I have found to be a powerful predisposing cause of nterine inflammation, owing probably to the intensity of the mollimen hæmorrhagicum, and to the length of time during which it persists, during which the patient is exposed to many perturbing causes. The intensity of the physiological congestion is evidenced by the fact, that for one, two, or three days before and after menstruation these females often have a slight white or leucorrhceal discharge, even when in perfect health.

With many females the first manifestation of the menses is unaccompanied by pain. The menstrual flux makes its appearance with scarcely any previous admonition of its advent, and continues to appear without pain or uneasiness; or if pain is present, it is slight and limited to the first few hours. This is the most favourable mode in which the menstrual function can take place, and the one which affords the greatest guarantee of future immunity from inflammatory disease. It is, however, by no means the rule; with many women, the first advent and the sub-

* Essays and Notes on the Physiology and Diseases of Women. 1851. sequent appearance of the menses, are attended, physiologically, throughout life, with great uterine pain. With some the pain is limited to the first few hours, with others it exists for a shorter or longer period before, and lasts throughout, the period.

The periodicity of menstruation also varies physiologically to a great extent. I have found that four weeks or twenty-eight days, the lunar month, is the most general term; but the periodical return of the menses may take place at any time between the third and the fifth. Most authors allow even a greater latitude; but I believe that the constant return of the menses at an earlier or later period will nearly always be found, on a careful inquiry, to be a pathological symptom, and to be connected with local disease.

From what precedes, it will be perceived that the physiological variations of menstruation - variations quite compatible with health-are so numerous and so great, that it is impossible to lay down any standard by which the integrity of the function can be generally tested. The above fact would much diminish the importance of the changes that occur in the menstrual function in disease, as an element of diagnosis, were it not that this irregularity is not observed, physiologically, in each individual case. In other words, every female has her own individual standard, to which she generally remains true throughout her life, unless the uterine organs be the seat of disease, or the gereral health be deeply modified by some other cause. Once, therefore, we have ascertained the mode in which menstruation occurs in any particular female, at an epoch when it may be fairly presumed that she was in good uterine health, we are authorized to surmise the presence of uterine or ovarian diseaseand, geverally speaking, the former-if any marked and permanent change takes place.

It is the ignorance of this important fact that has filled with errors, as I have already stated, all existing treatises on menstruation, at nearly every page of which are narrated, as physiological, cases which $I$ at once recognise as most decidedly pathological. This circumstance, therefore, must greatly invalidate the valueof the conclusions at which these authors have arrived, whether statistical or otherwise, with respect to the physiology of menstruation.

Cambridge-square, Hyde-park, 1851.

$$
\text { (To be continued.) }
$$

\section{CASE OF SUICIDAL WOUND OF THE THROAT.} WITH REMARES.

BY A UGUSTUS EVES, M.D. \& F.R.C.S. SURGEON TO THE CHELTENHAM GENRRAL HOSFITAL.

In The Lancex for May 26, 1849, I published a case of suicidal wound of the throat, successfully treated by ligature of the common carotid artery. In my remarks attached to the case, some points of practice were discussed. As the following case illustrates some of these points, I will take the liberty of relating it, and then deduce those pathological and practical suggestions which it is calculated to afford.

C. $\mathrm{P}-$ aged about thirty, admitted into the Cheltenham Hospital, July 28th, 1850, with a large transverse, gaping wound in the neck, inflicted by himself with a carving-knife. The larynx was completely divided, the incision passing between the thyroid and cricoid cartilages, which were separated to a great extent; no important artery appeared to be injured. He had been suffering from some affection of the brain, and was still in a state of partial insanity. Mr. Hartley placed sutures through the integuments, and brought the wound together with adhesive plaster.

July 29 th.-Before the nurse could prevent him he rose from his bed, and tore the wound open. A straight-waistcoat was put on him, and he had the following draught: Potassiotartrate of antimony, half a grain; Battley's solution, twenty minims; water, one ounce.

30th.-Having been out of Cheltenham, I saw him for the first time to-day. The cartilages of the larynx were widely separated, and so movable as to lead to the supposition that recovery could not take place unless by some means they could be approximated and kept in apposition. I again brought the integuments together with sutures and adhesive plaster, and endeavoured, by bandages, to confine the chin in the direction of the chest. To take forty minims of tincture of opium at night. He was ordered not to take nourishment by the mouth, but to have a pint of beef-tea as an injection twice a day.

Aug. 1.-Wound in position; sleepless nights. Ordered, 
hydrochlorate of morphia, half a grain; water, one ounce; to be taken at night.

4th.- Yesterday he vomited a great quantity of blood, some passing through the wound. The sutures have again given way. The attendants have allowed him to drink a small quantity of water, which passes out at the wound; so also did the medicine, which was administered in the smallest possible form, that the parts might not be necessarily disturbed by the action of swallowing. The following. injection to be given to-night: Tincture of opium, forty minims; mucilage, two drachms; water, six ounces.

6th.-He has had venous hæmorrhage several times to a great extent; no vessel could be observed from whence the bleeding arose. Thinking the patient had but a small chance of recovery unless the cartilages were kept in apposition, today I passed two sutures through the cricoid and thyroid cartilages, by which means they were bronght completely in contact. The integuments were approximated as much as possible by adhesive plaster, and the head flexed forward by bandages. He is to take a small quantity of milk.

7th.-To-day the sutures cut through the cricoid cartilage; the thyroid is completely exposed throughout its whole inferior anterior margin, and very movable, yet the wound is filling up by granulations. The wound was dressed by applying a broad strap of plaster under it, and bringing the ends up behind the ears. To have milk and eggs.

9th.- To-day he conld speak and swallow; has been very quiet.

15 th. - The wound is filling up by means of very large granulations; the cartilages have not united, neither have their cut edges thrown out granulations, but these have arisen from the surrounding parts to a very great extent, thereby blocking up the opening between the cartilages, and in some degree forcing them together. He eats and speaks well.

Sept. 9th.-Wound completely filled up by granulations, which have become consolidated over the division of the cartilages; external wound very small. He has had symptoms of insanity occasionally throughout the treatment, which, by producing restlessness and motion, very much interfered with that quiescent state of the parts so necessary to his cure by the means first attempted. The straight waistcoat has been used every night until this date. This, so far from being disagreeable to him, had an effect evidently soothing, and he regularly asked for its application before composing himself to rest for the night. Hydrochlorate of morphia was also administered occasionally.

About the end of September, the partial insanity still continuing, he was sent to the Gloucester County Asylum. At the present time, December, 1851, I hear he has quite recovered, and has left the asylum.

Remarks.- Several points of interest present themselves to our notice in this case. Firstly, the remarkable power exerted by the constitution in healing this very large wound. Judging $\dot{a}$ priori, I should not have supposed this fact at all probable, unless the parts conld be kept in complete apposition, which was not the case. Again, the manner in which this process was accomplished is worthy of notice: the cartilages did not unite in any way, but exceedingly large granulations arose from the surrounding parts, and blocked up the opening; these loose and large granulations afterwards contracted and became firm and consolidated; the necessity for speedily closing the aperture appearing, as it were, the cause of such unusually large granulations.

Another point of interest is the probable wound of the cosophagus, in this case. It has been supposed by some that it were almost impossible to wound the cesophagus without inflicting a wound which, by dividing the carotids, internal jugular veins, and pneumogastric nerves, would be instantly fatal. But Mr. Porter states that he " met with a patient who contrived, with only a blunt and rusty penknife, to divide the windpipe and cesophagus down to the spine, without wounding a large vessel, and he lived for three days after the infliction of so dreadful an injury," (Porter on Larynx, \&c., p. 242). I am well aware, however, that food passing through a wound in the neck, is no evidence of itself of an aperture in the cesophagus; in common cases of suicidal wounds in this part, the incision being above the thyroid cartilage, communicates with the mouth. But it is also well-ascertained that in wounds of the trachea or larynx below the thyroid cartilage, liquids taken into the mouth, will pass out through the wound, without any aperture being present in the œsophagus. A case of this kind, authenticated by dissection, is related by Mr. Porter (p. 168). This is attributed to the epiglottis ceasing to perform its function of shutting the glottis. It is, however, taking a very limited view of this subject, to suppose that closure of the glottis depends alone upon the epiglottis.

Mr. Mayo (Physiology, 3rd edition, p. 360) had a patient in the Middlesex Hospital, so wounded by a suicidal attempt, that he could look into the upper part of the larynx, and observe what took place when the patient voluntarily closed the passage through it; " the arytænoids were closely pressed together, and the upper ligaments of the glottis or the walls of the ventricle seemed brought into contact."

According to Mayo, Magendie first made the observation, that the epiglottis is not the protector of the larynx against the passage of food into the windpipe during deglutition. He found that that part might be removed from dogs, and that they retained perfectly the power of swallowing; while, on the other hand, the epiglottis being left entire, if the upper laryngeal nerve were divided, the attempt to swallow invariably brought on fits of choking, from the food finding its way down the windpipe. The superior laryngeal nerve, then, appears to supply that acute sensibility with which the glottis is endowed as a guard against the ingress of foreign bodies. Any impressions made on the filaments of this nerve are conveyed to the medulla oblongata, from whence they are reflected to the filaments of the recurrent laryngeal, (for the most part a motor nerve, and excite contraction of the muscles that close the glottis. Hence it follows that injury of any considerable filaments of either of these nerves may so derange the mechanism of the glottis as to allow liquids to pass down into the trachea, and out at a wound thereot, with. out any direct communication with the cesophagus. A practical fact is connected with these remarks, which is, that a wound or injury of the epiglottis is not so formidable as it would be if that organ were the only guard of the glottis. But to return to the question. Was the csophagus wonnded in the case I have related? I think it was, because I account for the vomiting of blood by supposing that this fluid first passed down into the stomach through an opening in the œsophagus.

Another point worthy of remark in this case is the possession of the voice concomitant with a large wound in the larynx. In my notes, I have noticed that the man was able to speak on the 9 th of August, the twelfth day after the suicidal attempt was made; but my friend, Mr. Hartley, the resident medical officer of the hospital, states that this was the case when the patient was first brought into the hospital, whether before or after dressing the wound, he cannot recollect; but in another case of a woman, who inflicted a fatal wound on the trachea, dividing it to a great extent between the thyroid and cricoid cartilages, he states that the voice remained, and the loud cries of the unfortunate patient were heard at a great distance. I have mentioned this point on the authority of Mr. Hartley, as it might be of interest in legal medicine, especially as in the excellent work of Dr. Taylor it is stated - "The fact is well known medically, that when the trachea is divided, the voice is lost," (Medical Jurisprudence, 3rd edition, p. 329.) With regard to this matter of the voice, I think the case is very clearly stated in the Bibliothèque du Médecin-Praticien, (tome douzième) p. 44. Speaking of wounds of the larynx, it is stated, that if they are situated below or carried through the vocal cords, the voice is lost, or at all events altered proportionally to the extent of the wound. This suppression of the voice especially takes place when, in a complete transverse division of the larynx or trachea, the head, in throwing itself backwards, separates one from the other of the two extremities of the air-tube. In bending the neck forwards, the divided extremities are again brought together; the continuity of the tube is established, and the voice returns. Reference is then made to a well-known cave is a wounded person, who at first could not speak, but, after the illustrious Ambrose Paré had bent the neck forwards, was able to give an account of his accident. The voice continues lost as long as this condition remains unaccomplished. One exception to this is cited-viz., a case recorded in the Journal Hebdomadaire, tome iii.:-A galleyslave had completely divided the larynx below the vocal cords; the upper part of this organ was obliterated; the lower part communicated with the pharynx; the skin of the neck was united to the anterior wall of the last-mentioned organ, and yet the man still was able to speak.

The practical deductions which may be added to the pathological suggestions from this case, are, that in cases apparently hopeless we may hope much from the natural powers if the constitution be good; that the employment of sutures through the cartilages may be had recourse to without creating that 
degree of irritation of which some authors speak, and therefore they ought to be employed as a means of bringing the divided parts of the larynx together; that every means to procure quietude and approximation of the wounded parts should be most carefully employed. Should I again have to bring the cartilages of the larynx in apposition, I would use elastic india-rubber sutures, which, by giving way to the movements of swallowing and respiration, would not so readily tear through the cartilages as silk-ligatures. I would here beg to reiterate the advice given in my last paper on this subject, that the patient for the first three or four days should be supported freely by strong nourishing injections; and as opium is generally an essential in the treatment, it may be given in the same way.

Cheltenham, December, 1851.

\section{OBSERVATIONS ON DISEASES OF THE EAR; WITH PRACTICAL REMARKS ON THEIR VARIETIES AND TREATMENT.}

Br THOMAS BARRETT, Esq., M.R.C.S., L.S.A., \&c. SURGEON TO THE EAR AND EYE INFIRAARY, BATH.

\section{No. $I$.}

IT must be, indeed, a matter of regret to all who think upon the subject, that the knowledge of diseases of the ear is very far behind that of other organs of the body ; and there can be no doubt that, as a consequence, a large proportion of diseases of this most important organ, treated at random, or at any rate on anything but sound pathological principles, must end in results as unsatisfactory to the practitioner as disastrous to the patient.

Surely no one will deny that the care and attention to the condition of an organ on which so much of our comfort, and happiness, and enjoyment depend, should be a legitimate and proper object of the student of medicine; and yet, in the absence of special lectures, or clinical instructions, or even the requirements of the knowledge at examinations before public boards, he is disposed to look at it as a supplemental portion of medical knowledge, of little value, or little importance.

The present medical session has commenced; the various lec tures and clinical instructions are fully advertised; but a course of lectures on aural surgery does not show itself among the list; and whilst institutions for the relief of nearly every other malady that the human frame can be subject to, are prominently brought forward, and are nobly and liberally supported, both in Iondon and throughout the provinces, there are not in the kingdom twenty institutions devoted to the treatment of ear diseases.

The subject has been brought before the profession, and records of cases, fe indeed, but most valuable, have appeared occasionally in the public journals. I am desirous of avaling myself of the opportunity which my connexion with a public institution has given me, of studying and treating these important diseases, by selecting from my note-book such cases of diseases of the ear as will tend to illustrate the variety of disease, the treatment, and results.

\section{Acute Myringitis, with perforation of Membrana Tympani.}

Case 1.-The Rer. J. H-, aged forty.five, on the 2nd March, 1850, performed the burial service in a damp country churchyard, and in the same evening felt he had "taken cold." He was attacked during the night with great pain in the left side of the face, extending into the ear, which increased and was followed by difficulty of swallowing and tenderness round the angle of the jaw, and over the mastoid process; all which symptoms, as he had previously suffered from neuralgia and rheumatism, he attributed to these causes, and took for his relief large doses of quinine and opium. The pains and discomfort continued to increase, and he began to experience deafness and confused sounds in the ear, with great irritation over the whole of the auricle, and extending far in to the ear-passage.

This alarmed him, and on the 9 th March he consulted me. I found him then labouring under considerable pain, as before mentioned, penetrating deeply into the head, and increased by every effort to swallow or open the mouth wide. He feels the carotid of that side beating loudly in the ear; has timnitus; the noise, he says, at one time is like the scraping of a saw-mill-at another like the rushing of wind. He does not fancy he is very deaf, but $I$ find the hearing distance is less than three inches. I take this opportunity of mentioning that, with a view to ascertain and note carefully the variation in the hearing power of patients, whilst under treatment, I always employ a metronome for this purpose; its steady, d:stinct, and uniform beat is more to be depended upon, and therefore is a much better and surer test, than the ticking of a watch as ordinarily used.

An examination of the ear showed the auricle in a state of phlegmonous inflammation, and the tragus and commencement of the ear-passage so swollen that no inspection could be made by the speculum.

The next dar, after free leeching over the mastoid process, and in front of the auricle, and the application of warm fomentations and bran poultices, I was enabled to examine the condition of the ear-passage and membrana tympani.

The whole length of the canal was covered with a muco. purulent secretion. On this being washed away with warm water, the canal was found tender to the touch, swollen, and very red. The membrana tympani, from its excessive vascularity, was bright rose colour; the projection of the malleus could not be detected; its whole surface looked pulpy and villous. The tonsils on both sides were enlarged and slightly ulcerated; the fauces generally relaxed; and no air could be passed through the Eustachian tube on the affected side, the effort to do so being attended with great pain. There is a good deal of constitutional disturbance. The appetite is bad, and the night sleepless. Leeches in front of the meatus were again ordered, and counterirritation to be freely kept up over the mastoid process. A pill, composed of extract of hemlock and colchicum, and bluempill, was directed to be taken three times a-day.

March 13.-The pain and irritation in the ear, and diffculty of swallowing, had considerably lessened; there is otorrhœa. The noises in the ear continue most distressing. He is now able to force air through the Eustachian tube; the otoscope detects the air as gurgling through a mucous fluid, and with a peculiar hissing sound indicative of a rupture of the membrane. The speculum shows the membrana tympani still deeply red, though darker in colour, with two distinct vesicles on its surface, though so swollen and pulpy that the exact situation of the perforation could not be detected. The hearing distance is not an inch; indeed, the deafness is nearly complete. The medicine, and counter-irritation over the mastoid process, were ordered to be continued.

16th.-The constitutional symptoms much improved; the pain and irritation in the ear and the adjacent parts are lessened; the noise he likens now to the lowing of cattle. The discharge is rery profuse; no improvement in hearing.

The ear was washed out with warm water, and I found a polypous excrescence had shot out from the interior wall of the canal and completely filled up the passage. I removed it by means of Mr. Wilde's ingenious and very useful "snare," and found the membrana tympani of a pale pink colour, with very little remains of its former villous and pulpy appearance. The perforation could not be seen; it was of au irregular shape, and situated near the anterior and inferior portion of the membrane. The removal of the fungus extended the hearing distance at once to about four inches.

As the mouth was rather tender, the pills were discontinued, and the iodide of potassium in bitter infusion was directed to be taken three times a day. A fine point of nitrate of silver was applied very lightly to the edge of the perforation, and gentle syringing with a weak tannin solution ordered twice a day.

This patient continued improving under treatment, both in his general health and in the local malady. On the 15th April, I found the membrana tympani rather thick, and studded with opaque spots ; there is slight discharge; the hearing distance is about one foot; the tinnitus still continues. Counter-irritation behind the ear with tartrate-of-antimony ointment; the occasional pencilling of the membrane and auditory canal with a solution of nitrace of silver (five grains to one ounce); and the Bath waters, formed the local and constitutional treatment for another month. At the end of that time the general health was perfectly restored; the discharge had quite ceased : there is still some tinnitus, and occasionally loud crackings, on gaping or swallowing, are heard by him.

I may observe that this gentleman has suffered a long time from dysphonia clericorum; the throat is always more or less relaxed, and the Eustachian tube, I doubt not, partakes of the same irritation of the membrane. The perforation in the tympanal membrane is of an irregular shape, occupying about a fifth of the membrane, the remaining portion of which has now assumed a tolerably healthy appearance; the auditory canal is dry, and without any secretion of cerumen. The hearing distance is about two feet.

I considered this a favourable case for the use of the hydrated cotton, as recommended by $\mathrm{Mr}$. Yearsley, and to the value of which, in judiciously selected cases, and when applied with due care and tact, I have before borne my full testimony.

Its application increased directly the hearing distance to nearly three yards. 